\title{
How can LVAD support influence ventricular energetics parameters in advanced heart failure patients? A retrospective study.
}

\author{
Claudio De Lazzari ${ }^{1}$ \\ National Research Council, Institute of Clinical Physiology (IFC-CNR), Rome, Italy \\ Via Palestro, 32 (00185) Rome, Italy \\ National Institute for Cardiovascular Research (I.N.R.C.), Bologna, Italy \\ Via Irnerio, 48 (40126) Bologna, Italy \\ claudio.delazzari@ifc.cnr.it.
}

\author{
Massimo Capoccia \\ Royal Brompton Hospital, Royal Brompton \& Harefield NHS Foundation Trust, UK \\ Department of Biomedical Engineering, University of Strathclyde, Glasgow, UK \\ capoccia@doctors.org.uk
}

\author{
Silvia Marconi \\ National Research Council, Institute of Clinical Physiology (IFC-CNR), Rome, Italy \\ Via Palestro, 32 (00185) Rome, Italy \\ silvia.marconi@ifc.cnr.it
}

\footnotetext{
${ }^{1}$ Corresponding author:

Dr. Eng. Claudio De Lazzari

C.N.R., Institute of Clinical Physiology, Rome, Italy

Via Palestro, 32

00185 Rome, Italy

Fax: $\quad+390649936299$

E-mail: claudio.delazzari@ifc.cnr.it
} 
De Lazzari Claudio

\section{ABSTRACT}

Background and Objective: Here we present a retrospective analysis of six heart failure patients previously discussed at a multidisciplinary team meeting. Only three out of six patients underwent LVAD insertion as the most appropriate management option. Methods: We sought to reproduce the baseline conditions of these patients on hospital admission using our cardiovascular software simulator (CARDIOSIM ${ }^{\odot}$ ). Subsequently, we simulated the effects of LVAD support and drug administration on left and right ventricular energetics parameters. LVAD assistance was delivered by CARDIOSIM ${ }^{\odot}$ based on the module reproducing the behavior of the Berlin Heart INCOR pump.

Results: The results of our simulations were in agreement with the multidisciplinary team meeting outcome. The analysis of ventricular energetics parameters based on external work and pressure volume area confirmed LVAD support as a beneficial therapeutic option for the three patients considered eligible for this type of treatment. The effects induced by LVAD support and drugs administration showed specific patterns between the two groups of patients.

Conclusion: A quantitative approach with the ability to predict outcome during patient's assessment may well be an aid and not a substitute for clinical decision-making.

Key words: Heart failure; External work; Pressure volume area; Software simulation; LVAD; Milrinone. 
De Lazzari Claudio

\section{INTRODUCTION}

Patient-specific modelling has received particular attention in recent years in view of its potential to tailor the most suitable treatment according to the group of patients considered [1]. The success of this approach would largely depend on the method used and its suitability within the constraints of the clinical environment. Although 3D modelling based on imaging reconstruction from CT-scan is an attractive prospect, the high computational power required for a complete haemodynamic analysis remains the main limitation. Nevertheless, this method would be more suitable for long-term planning in elective cases. Pressure-volume analysis of cardiovascular function is a powerful approach that has been mainly confined to a research environment although its application to a clinical setting has been long recognized but not completely accepted [2]. Lumped parameter modelling gives a simplified and effective description of the cardiovascular system during a healthy and diseased status. A modified time-varying elastance is appropriate in this context given the criticism to the original concept when applied to a mechanically supported left ventricle [3]. The aim of our project is to introduce a different approach to the clinical environment based on modelling and simulation for optimization of device treatment and outcome prediction. In other words, a system based on a more quantitative approach and not a substitute for clinical experience: the clinician remains the ultimate decision-maker based on the available data. The software used for the analysis has been developed and refined by our group over the years in order to be ready for applications based on real life clinical scenarios. The ultimate hurdle was to prove the ability of the software to reproduce accurately and 
De Lazzari Claudio

reliably clinical data obtained from echocardiographic and right heart catheter assessment which would be used to simulate baseline conditions and the potential outcome in order to optimize and select the most suitable treatment for different group of patients. Advanced heart failure patients are complex and demanding requiring a significant amount of investigations and data. Particularly, a group of them had generated heated discussion during MDT meetings (MC). Therefore, we considered this group of six patients as an ideal pilot study that may well generate further research and possibly a prospective comparison in a non distant future.

In previous work, we have addressed the issues related to the preoperative assessment of patients in advanced heart failure and argued the potential of a simulation approach in the clinical decision-making process with a view to outcome prediction and optimization of device treatment. Our efforts mainly focused on the hemodynamic parameters derived from echocardiographic assessment and right heart catheter [4-6].

CARDIOSIM $^{\odot}$ is the numerical simulator of the cardiovascular system used for the study [7-15]. This software is based on 0-D mathematical models, which reproduce the ventricular, atrial and septal behavior according to a modified time-varying elastance concept $[4,7,8]$. The library of the simulator allows the assembly of specific modules describing different circulatory districts [8-15].

We have already reproduced the baseline hemodynamic conditions of advanced heart failure patients on hospital admission in recently published work. Subsequently, we simulated the effects induced on hemodynamic parameters by left ventricular assist 
De Lazzari Claudio

device (LVAD) support and by Milrinone administration. LVAD assistance was delivered by CARDIOSIM ${ }^{\odot}$ module reproducing the behavior of the Berlin Heart INCOR Pump [4, 5], which is a continuous flow pump specifically designed for long-term support but also suitable as a bridge to transplant and bridge to recovery.

In the present work, we have considered both a hemodynamic and energetics analysis of the effects induced by LVAD support and drug administration according to the previously used approach. We have analyzed the baseline conditions on hospital admission and following LVAD support (with or without Milrinone administration) for each patient in terms of left and right ventricular external work $(E W)$ and pressure volume area (PVA) using CARDIOSIM ${ }^{\circledR}[10,12,13]$. Then, we have compared the measured and the simulated parameters by statistical analysis with Stata ${ }^{\circledR}$ using Student's t-test and Mann-Whitney test to evaluate the accuracy and reliability of the simulations.

\section{MATERIAL AND METHOD}

\section{Cardiovascular and Berlin Heart INCOR pump numerical models}

The cardiovascular network configuration used to simulate the baseline hemodynamic conditions on hospital admission for the six patients included in this study is represented in Fig. 1. The network consists of left and right heart, systemic arterial and venous sections, main, small, arteriole, capillary and venous pulmonary sections. In addition, an electrical analogue of LVAD is showed in Fig. 1. The behavior of both native ventricles was implemented using a modified time-varying elastance theory. Also the inter-ventricular septum (IVS) activity was described by the time-varying elastance 
De Lazzari Claudio

model $[4,6,16]$. Based on these assumptions, the following equations described the IVS interaction and the instantaneous left and right ventricular pressure:

$$
\left\{\begin{array}{l}
P_{l v}(t)=\left[\frac{e_{V s p}(t) \cdot e_{l v}(t)}{e_{l v}(t)+e_{V s p}(t)}\right] \cdot\left[V_{l v}(t)-V_{l v, 0}\right]+\left[\frac{e_{l v}(t)}{e_{l v}(t)+e_{V s p}(t)}\right] \cdot P_{r v}(t)+\left[\frac{e_{V s p}(t)}{e_{l v}(t)+e_{V s p}(t)}\right] \cdot P_{l v, 0} \\
P_{r v}(t)=\left[\frac{e_{V s p}(t) \cdot e_{r v}(t)}{e_{V s p}(t)+e_{r v}(t)}\right] \cdot\left[V_{r v}(t)-V_{r v, 0}\right]+\left[\frac{e_{r v}(t)}{e_{V s p}(t)+e_{r v}(t)}\right] \cdot P_{l v}(t)+\left[\frac{e_{V s p}(t)}{e_{V s p}(t)+e_{r v}(t)}\right] \cdot P_{r v, 0}
\end{array}\right.
$$

In the same way, both the native atrial and the inter-atrial septum (IAS) behavior were implemented applying the time-varying elastance concept; consequently, the IAS interaction and the left and right instantaneous atrial pressure were described by $[4,6$, 16]:

$$
\left\{\begin{array}{l}
P_{l a}(t)=\left[\frac{e_{A s p}(t) \cdot e_{l a}(t)}{e_{l a}(t)+e_{A s p}(t)}\right] \cdot\left[V_{l a}(t)-V_{l a, 0}\right]+\left[\frac{e_{l a}(t)}{e_{l a}(t)+e_{A s p}(t)}\right] \cdot P_{r a}(t)+\left[\frac{e_{A s p}(t)}{e_{l a}(t)+e_{A s p}(t)}\right] \cdot P_{l a, 0} \\
P_{r a}(t)=\left[\frac{e_{A s p}(t) \cdot e_{r a}(t)}{e_{A s p}(t)+e_{r a}(t)}\right] \cdot\left[V_{r a}(t)-V_{r a, 0}\right]+\left[\frac{e_{r a}(t)}{e_{A s p}(t)+e_{r a}(t)}\right] \cdot P_{l a}(t)+\left[\frac{e_{A s p}(t)}{e_{A s p}(t)+e_{r a}(t)}\right] \cdot P_{r a, 0}
\end{array}\right.
$$

Table 1 shows the symbols used in Eqs. (1) and (2).

The software used in this work can be reproduced applying Ohm's law, Kirchhoff's circuit laws and Eqs. (1) and (2) to resolve the cardiovascular network (Fig. 1). The INCOR pump when applied as LVAD takes blood from the left ventricle and ejects it into the aorta (Fig. 1). The rotor pump produces a maximum flow rate of $5 \mathrm{~L} / \mathrm{min}$ with a 
De Lazzari Claudio

rotational speed between 5000 and 10000 rpm. The continuous blood flow can be modelled as $[4,5,17,18]$ :

$$
\begin{aligned}
& Q_{L V A D}=A_{L V A D, 0}+\omega \cdot A_{L V A D, 1}+A_{L V A D, 2} \cdot\left(P_{L V A D_{\text {OUT }}}-P_{L V A D_{I N}}\right)+A_{L V A D, 3} \cdot \omega \cdot\left(P_{L V A D_{\text {OUT }}}-P_{L V A D_{I N}}\right)+ \\
& +A_{L V A D, 4} \cdot \omega^{2} \cdot\left(P_{L V A D_{\text {OUT }}}-P_{L V A D_{I N}}\right)+A_{L V A D, 5} \cdot \omega \cdot\left(P_{L V A D_{\text {OUT }}}-P_{L V A D_{I N}}\right)^{2}+A_{L V A D, 6} \cdot \omega^{2} \cdot\left(P_{L V A D_{\text {OUT }}}-P_{L_{V A D} D_{I N}}\right)^{2}
\end{aligned}
$$

The INCOR pump behavior is reproduced by solving the network reported in Fig.1 and using Eq. (3).

Table 2 shows symbols and parameter values used during the simulations. After manual insertion of heart rate $(H R)$, mean arterial blood pressure $(B P)$, stroke volume $(S V)$, left ventricular ejection fraction ( $\left.E F_{L E F T}\right)$ and body surface area $(B S A)$, CARDIOSIM $^{\odot}$ software estimates end-diastolic volume (EDV), end-systolic volume (ESV) and the slope $\left(E_{e s}\right)$ of left ventricular end-systolic pressure volume relationship (ESPVR). This task allows placement of the left ventricular loop in the pressure-volume (P-V) plane to reproduce the baseline conditions of each patient (Fig. 2). The simulated mean left atrial pressure (which can be considered equivalent to the measured capillary wedge pressure - $P C W P$ ) allows $\mathrm{CARDIOSIM}^{\odot}$ software to reproduce left ventricular filling.

\section{Heart Failure Patients}

The retrospective analysis was performed starting with hemodynamic data measured in the six patients (Table 3). Patients \#1, \#2, and \#3 had been described in previous work [4-6]. Patient \#4 was diagnosed with dilated cardiomyopathy, patients \#5 and \#6 were diagnosed with ischaemic cardiomyopathy. 
De Lazzari Claudio

Hemodynamic data were measured using right heart catheterization (RHC).

\section{Experimental Method}

Firstly, we reproduced the baseline conditions for each patient on hospital admission using the cardiovascular simulator in order to evaluate left and right external work and pressure volume area [19-22]. Secondly, the Berlin Heart INCOR pump delivered assistance according to different settings:

1] for the first three patients, the pump was driven with a rotational speed of $6000 \mathrm{rpm}$ to obtain the best hemodynamic conditions. Then, the effect of Milrinone administration [4-6, 23] was simulated during LVAD assistance [24]. Milrinone is a phosphodiesterase inhibitor, which enhances cardiac contractility by increasing intracellular levels of cyclic adenosine monophosphate (c-AMP). The drug is a positive inotropic and vasodilator agent used in the intensive care setting for short-term treatment of severe congestive heart failure unresponsive to conventional maintenance therapy, and for the treatment of patients with acute heart failure, including low output states following cardiac surgery [25];

2] for patients \#4 and \#5, the simulations included the analysis of the effects induced by continuous LVAD assistance (with and without Milrinone administration) with a rotational speed of 6000 and $8900 \mathrm{rpm}$;

3] for patient \#6, the simulations included the analysis of the effects of continuous LVAD assistance (with and without Milrinone administration) with a rotational speed of 8900 and 10000 rpm. 
De Lazzari Claudio

For patients \#1, \#2 and \#3, left and right ventricular EW and PVA were estimated by the cardiovascular simulator. For patients \#4, \#5 and \#6, the simulations aimed to obtain the best hemodynamic conditions and evaluate left and right ventricular EW and PVA (Fig.2).

Finally, the accuracy and reliability of the cardiovascular software simulator assembled as described in $[4,5]$ was evaluated with the program Stata ${ }^{\circledR}$ using Student's t-test and Mann Whitney test in view of the multi-parametric nature of the software [26]. Some of the measured and simulated baseline parameters were compared and a pvalue $<0.05$ was considered statistically significant. The $p$-value from Student's t-test was considered when the two tests agreed; discordance between the two tests would warrant the $p$-value from Mann-Whitney test because more accurate given its nonparametric nature.

\section{RESULTS}

Table 4 shows the simulation results of the hemodynamic parameters measured in the six patients. The $E_{\alpha} / E_{\text {es }}$ ratio describes the interaction between the left ventricle and the arterial system, namely ventricular-arterial coupling, which is an index of global cardiovascular efficiency.

Table 5 shows the effects on hemodynamic parameters induced by simultaneous LVAD assistance and Milrinone administration in patients \#4, \#5 and \#6 with a rotational speed of $8900 \mathrm{rpm}$. 
Figure 3 shows the effects on left ventricular EW induced by LVAD assistance with or without Milrinone administration in the six patients. The upper panel shows the percentage variation of EW compared to baseline (admission) conditions in patients \#1, \#2 and \#3 during LVAD support with and without drug administration. The pump rotational speed was fixed at $6000 \mathrm{rpm}$, which gave the best hemodynamic conditions $[4,5]$. In all three patients, LVAD support reduced left ventricular $E W$ by values ranging from $9 \%$ to $14 \%$ compared to non-assisted conditions. Concomitant LVAD support and Milrinone administration produced an opposite effect on left ventricular $E W$ with particular reference to patients \#1 and \#3 where $E W$ increased compared to baseline (admission) conditions. This effect may be related to improved ventricular contractility following drug administration. Left ventricular EW percent variation compared to baseline conditions calculated for patients \#4, \#5 and \#6 are listed in the lower panel of Fig. 3. The LVAD rotational speed remained at 6000,8900 and $10000 \mathrm{rpm}$ for patient \#4 during simulations with drug administration. A pump rotational speed of 8900 and 10000 rpm was considered in patients \#5 and \#6. Concomitant LVAD support at 6000 rpm with Milrinone administration was not carried out for patient \#6. Left ventricular assistance caused a reduction in left ventricular $E W$ in all these patients, with particular reference to patients \#4 and \#6 where an EW reduction up to $70 \%$ compared to baseline was observed. Simulations with INCOR pump and Milrinone showed an EW percent variation between $30 \%$ and $63 \%$ in all patients.

LVAD support alone did not influence right ventricular EW significantly in all patients (Fig. 4). Simulations with LVAD support and drug administration showed an 
De Lazzari Claudio

appreciable increase in right ventricular $E W$ in patients $\# 1, \# 2, \# 3, \# 4$ and $\# 6$ regardless of the pump rotational speed.

Figure 5 shows the percent variation of left ventricular pressure volume area compared to control conditions. The upper panel shows PVA variation of patients \#1, \#2 and \#3 during LVAD support with a rotational speed of $6000 \mathrm{rpm}$ and Milrinone administration. The simulations showed PVA reduction in patient \#3 by $30 \%$ in both cases. Simulations with a pump rotational speed at $6000 \mathrm{rpm}$ produced the best hemodynamic conditions for these three patients $[4,5]$. The results for patients \#4, \#5 and \#6 are listed in the lower panel. In all cases during LVAD support alone, a PVA reduction from $30 \%$ to $60 \%$ compared to baseline conditions was observed. A lower reduction in PVA percent variation occurred during LVAD support at $10000 \mathrm{rpm}$ and Milrinone administration in patients \#4 and \#6. Figures 4, 5 and 6 show that the best mechanical circulatory support system (MCSS) achievable performance in terms of energetics parameters depends on the patient's baseline conditions following hospital admission [27-29] in line with the effect of preload on MCSS performance from a hemodynamic point of view $[29,30]$.

\section{STATISTICAL ANALYSIS}

Despite the limited number of patients considered, the statistical analysis gave encouraging and promising results about the accuracy and reliability of the simulations carried out. Here are some findings: 
De Lazzari Claudio

$\checkmark$ SBP (systolic blood pressure): the p-value from Student's t-test was 0.32 with test concordance confirming the accuracy of CARDIOSIM ${ }^{\odot}$ for this parameter.

$\checkmark D B P$ (diastolic blood pressure): the analysis using Mann-Whitney test showed a $\mathrm{p}$ value of 0.42 with test discordance showing a statistical significance consistent with the tendency of the diastolic blood pressure to a "normal distribution" among the population. An indefinitely increase of the sample size would lead to a very slight overestimation of the parameter.

$\checkmark R A$ (right atrial pressure): the $\mathrm{p}$-value from Mann-Whitney test was 0.01 confirming a slight underestimation of the parameter.

$\checkmark R V$ (right ventricular pressure): the p-value from Mann-Whitney test was 0.07 confirming again the accuracy of the parameter reproduced by the software.

$\checkmark P A$ and $P C W P$ (pulmonary arterial and pulmonary capillary wedge pressures): the $p$ value from Student's t-test was 0.73 for $P A$ whereas from Mann-Whitney test it was 0.29 for PCWP. Test concordance confirmed the accuracy of the parameters reproduced by the cardiovascular software simulator.

$\checkmark E F_{\text {LEFT }}$ (left ventricular ejection fraction): the $\mathrm{p}$-value from Student's t-test was 0.61 with test concordance confirming the accuracy of the parameter reproduced by CARDIOSIM $^{\odot}$. Figure 7 shows that measured and simulated values are almost identical for all six patients.

$\checkmark E D V$ and ESV (end-diastolic and end-systolic volumes): the p-value from Student's ttest was $0.66(0.48)$ with test concordance confirming once again the accuracy of the parameters reproduced by the software simulator. 


\section{DISCUSSION}

Integration of experimental and clinical data through acquisition and parameter estimation techniques is showing consistent performance towards the application of computational modelling and simulation into daily clinical practice [31]. Nevertheless, model accuracy and reliability remain key issues to consider before its applicability to a clinical setting. In other words, the aim is model verification by solving the equations appropriately and model validation by solving the right equations leading to increased peer acceptance and gap bridging between analysts, experimentalists and clinicians [32]. The level of accuracy for a model depends on its use. From an engineering point of view, absolute truth is not required. A statistically meaningful comparison between computational findings and experimental measurements in relation to model use may be sufficient taking into account sources of error and uncertainty [32]. Needless to say, diagnostic challenges and outcome prediction reflect a need for further development of modelling approaches and data analysis in the context of a dynamical interaction between the heart and the vascular system in relation to changes following disease with a view to better understanding and targeted therapeutic intervention. The difficulty remains the translation of modelling analysis into meaningful and helpful tools for assessment and decision-making in a clinical setting [31]. The cardiovascular simulator used in this study addresses this issue given its ability to reproduce measured data in an accurate manner leading to reliable simulations with great potential for device treatment optimization and outcome prediction. The statistical analysis was designed 
De Lazzari Claudio

and applied in such a way to take into account the small number of patients and the parametric nature of our software. The outcome went beyond our expectations and definitely gave the final proof that our software is fit for purpose. A combined approach based on 0-D models, pressure-volume analysis and modified time-varying elastance seems an appropriate tool with easy translation to clinical practice with emphasis on patient-specific modelling, which remains a key player to understand disease mechanism and optimize its treatment [33]. Although left ventricular ejection fraction remains an important parameter for diagnosis and management of heart failure patients, its limitations must be taken into account with particular reference to its preload and afterload dependence and its relation with risk factors such as hypertension, diabetes and renal failure. An integrative approach including left and right ventricular function, ventricular and atrial geometry and clinical parameters gives a more accurate evaluation of clinical status and risk prediction [34]. Our modelling and simulation approach reflects this line of thinking considering the significant relationship between form and function in heart failure $[35,36]$. We also advocate the inclusion of the $E_{a} / E_{e s}$ ratio as an additional non-invasive measurement of ventricular-arterial coupling and its relation to remodeling and prognosis in chronic heart failure [37]. Finally, an analysis from the energetic point of view may give additional information about the efficiency and reserve of a failing system and its implication in relation to treatment and prognosis. 
De Lazzari Claudio

\section{CONCLUSIONS}

Our software has shown consistent performance as far as accuracy is concerned leading to reliable simulations based on patient-specific modelling. This is an additional step towards a prospective study, which could compare patient selection for LVAD support during a multidisciplinary team (MDT) meeting with and without simulation with a view to further validate its role for treatment optimization in cardiovascular disease with particular reference to advanced heart failure. A quantitative approach based on modelling and simulation with the ability to predict outcome during patient's assessment may become an aid and not a substitute for clinical decision-making. 


\section{NOMENCLATURE}

\begin{tabular}{|c|c|}
\hline$B P$ & Blood pressure [mmHg] \\
\hline$B S A$ & Body surface area $\left[\mathrm{m}^{2}\right]$ \\
\hline c-AMP & Cyclic adenosine monophosphate \\
\hline $\mathrm{Cl}$ & Cardiac index $\left[\mathrm{L} / \mathrm{min} / \mathrm{m}^{2}\right]$ \\
\hline $\mathrm{CO}$ & Cardiac output [L/min] \\
\hline COVENTR & Left ventricular output flow [L/min] \\
\hline$D B P$ & Diastolic blood pressure [mmHg] \\
\hline$E_{a}$ & Arterial elastance $[\mathrm{mmHg} / \mathrm{ml}]$ \\
\hline$e_{A s p}$ & IAS elastance $[\mathrm{mmHg} / \mathrm{ml}]$ \\
\hline$e_{l a}$ & Left atrial elastance $[\mathrm{mmHg} / \mathrm{ml}]$ \\
\hline$e_{/ v}$ & Left ventricular elastance $[\mathrm{mmHg} / \mathrm{ml}]$ \\
\hline$E_{e s}$ & Slope of ESPVR $[\mathrm{mmHg} / \mathrm{ml}]$ \\
\hline$e_{r a}$ & Right atrial elastance $[\mathrm{mmHg} / \mathrm{ml}]$ \\
\hline$e_{r v}$ & Right ventricular elastance $[\mathrm{mmHg} / \mathrm{ml}]$ \\
\hline$e_{v s p}$ & IVS elastance $[\mathrm{mmHg} / \mathrm{ml}]$ \\
\hline$E D V$ & End-diastolic volume [ml] \\
\hline$E F_{L E F T}$ & Left ventricular ejection fraction \\
\hline$E S V$ & End-systolic volume [ml] \\
\hline EDPVR (ESPVR) & End-diastolic (end-systolic) pressure volume relationship \\
\hline EW & External work \\
\hline$H R$ & Heart rate $[\mathrm{bpm}]$ \\
\hline IAS & Inter-atrial septum \\
\hline IVS & Inter-ventricular septum \\
\hline LVAD & Left ventricular assist device \\
\hline MCSS & Mechanical circulatory support system \\
\hline MDT & Multidisciplinary team \\
\hline$P_{l a}$ & Left atrial pressure $[\mathrm{mmHg}]$ \\
\hline$P_{l a, 0}$ & Resting left atrial pressure $[\mathrm{mmHg}]$ \\
\hline$P_{l v}$ & Left ventricular pressure $[\mathrm{mmHg}]$ \\
\hline$P_{l v, 0}$ & Resting left ventricular pressure $[\mathrm{mmHg}]$ \\
\hline Pra & Right atrial pressure [mmHg] \\
\hline$P_{r a, 0}$ & Resting right atrial pressure $[\mathrm{mmHg}]$ \\
\hline$P_{r v}$ & Right ventricular pressure $[\mathrm{mmHg}]$ \\
\hline$P_{r v, 0}$ & Resting right ventricular pressure $[\mathrm{mmHg}]$ \\
\hline $\mathrm{P}-\mathrm{V}$ & Pressure-volume \\
\hline$P A$ & Pulmonary arterial pressure $[\mathrm{mmHg}]$ \\
\hline PCWP & Pulmonary capillary wedge pressure [mmHg] \\
\hline$P E$ & Potential energy \\
\hline $\mathrm{P}-\mathrm{V}$ & Pressure-volume \\
\hline Ped(Pes) & End-diastolic (end-systolic) ventricular pressure [mmHg] \\
\hline PVA & Pressure volume area \\
\hline
\end{tabular}


De Lazzari Claudio

$\begin{array}{ll}\text { PVR } & \text { Pulmonary vascular resistance }[\mathrm{mmHg} \cdot \mathrm{s} / \mathrm{ml}] \\ Q_{L V A D} & \text { LVAD flow [L/min] } \\ R A & \text { Right atrial pressure }[\mathrm{mmHg}] \\ \text { RHC } & \text { Right heart catheterization } \\ R V & \text { Right ventricular pressure }[\mathrm{mmHg}] \\ \text { RVSWI } & \text { Right ventricular stroke work index }\left[\mathrm{g} / \mathrm{m}^{2} / \mathrm{beat}\right] \\ S B P & \text { Systolic blood pressure }[\mathrm{mmHg}] \\ S V & \text { Stroke volume [ml] } \\ \text { TPG } & \text { Trans-pulmonary pressure gradient }[\mathrm{mmHg}] \\ V_{0} & \text { Zero pressure filling volume }[\mathrm{ml}] \\ V_{l a} & \text { Left atrial volume [ml] } \\ V_{l a, 0} & \text { Resting left atrial volume }[\mathrm{ml}] \\ V_{l v} & \text { Left ventricular volume [ml] } \\ V_{l v, 0} & \text { Resting left ventricular volume }[\mathrm{ml}] \\ V_{r a} & \text { Right atrial volume [ml] } \\ V_{r a, 0} & \text { Resting right atrial volume }[\mathrm{ml}] \\ V_{r v} & \text { Right ventricular volume }[\mathrm{ml}] \\ V_{r v, 0} & \text { Resting right ventricular volume }[\mathrm{ml}]\end{array}$


De Lazzari Claudio

\section{CONFLICT OF INTEREST}

None

\section{ACKNOWLEDGEMENTS}

This work was supported by the Italian Ministry of Education, University and Research (M.I.U.R.) Flagship InterOmics Project (cod. PB05). 


\section{REFERENCES}

[1] Taylor, C.A., Figueroa, C.A., 2009, "Patient-specific Modelling of Cardiovascular Mechanics," Annu. Rev. Biomed. Eng., 11, pp. 109-134.

[2] Doshi, D., Burkhoff, D., 2016, "Cardiovascular Simulation of Heart Failure. Pathophysiology and Therapeutics," J. Card. Fail., 22(4), pp. 303-311.

[3] Vandenberghe, S., Segers, P., Steendijk, P., Meyns, B., et al., 2006, “Modelling Ventricular Function during Cardiac Assist: Does Time-Varying Elastance Work?" ASAIO J., 52, pp.4-8.

[4] Capoccia, M., Marconi, S., Singh, S.A., et al., 2018, "Simulation as a preoperative planning approach in advanced heart failure patients. A retrospective clinical analysis," BioMedical Engineering OnLine, 17(1), pp. 52.

[5] Capoccia, M., Marconi, S. and De Lazzari, C., 2018, "Decision-making in advanced heart failure patients requiring LVAD insertion: Can preoperative simulation become the way forward? A case study," Journal of Biomedical Engineering and Informatics, 4(2), pp. 8-20.

[6] Capoccia, M., Singh, S.A., De Lazzari C., 2017, "The Role of Simulation for Preoperative Planning in Patients Requiring Mechanical Circulatory Support", Proc. of the European Medical and Biological Engineering Conference (EMBEC) and the NordicBaltic Conference on Biomedical Engineering and Medical Physics (NBC), H. Eskola et al., eds., Tampere, Finland, 65, pp. 559-562.

[7] Ferrari, G., De Lazzari, C., Mimmo, R., Tosti, G., Ambrosi, D., 1991, "A modular numerical model of the cardiovascular system for studying and training in the field of cardiovascular physiopathology," J. Biomed. Eng., 14, pp. 91-107.

[8] De Lazzari, C., 2012, "Interaction between the septum and the left (right) ventricular free wall in order to evaluate the effects on coronary blood flow: numerical simulation," Comput. Methods. Biomech. Biomed. Eng., 15(12), pp. 1359-1368.

[9] De Lazzari, C., Genuini, I., et al., 2014, "Interactive simulator for e-Learning environments: a teaching software for health care professionals," BioMedical Engineering OnLine, 13, pp. 172.

[10] De Lazzari, C., Darowski, M., Wolski, P., et al., 2005, "In vivo and simulation study of artificial ventilation effects on energetic variables in cardiosurgical patients," Methods of Information in Medicine, 44(1), pp.98-105. 
[11] De Lazzari, C., Darowski, et al., 2006, "Modelling in the study of interaction of Hemopump device and artificial ventilation," Computers in Biology and Medicine, 36(11), pp. 1235-1251.

[12] De Lazzari, C., Darowski, et al., 2006, "The impact of rotary blood pump in conjunction with mechanical ventilation on ventricular energetic parameters: Numerical Simulation," Methods Inf. Med., 45, pp. 574-583.

[13] De Lazzari, C., Darowski, M., Ferrari, G., Clemente, F., Guaragno, M., 2001, "Ventricular energetics during mechanical ventilation and intraaortic balloon pumping? computer simulation," Journal of medical engineering \& technology, 25(3), pp. 103-111.

[14] De Lazzari, C., Ferrari, G., 2007, "Right Ventricular Assistance by Continuous Flow Device. A Numerical Simulation," Methods Inf. Med., 46, pp. 530-537.

[15] Darowski, M., De Lazzari, C., Ferrari, G., Clemente, G., Guaragno, M., 1999, "The influence of simultaneous intra-aortic balloon pumping and mechanical ventilation on hemodynamic parameters-Numerical simulation," Frontiers of Medical and Biological Engineering, 9(2), pp.155-174.

[16] De Lazzari, B., Quatember, B., Mottaghy, K., De Lazzari, C., 2017, "Numerical Models of the Cardiovascular System: Interaction Between the Septum and the Left (Right) Ventricular and Atrial Free Wall," in Cardiovascular and Pulmonary Artificial Organs: Educational Training Simulators. National Research Council Press, Rome, IT, Chap. 2. ISBN: 978-88-8080-240-2.

[17] Shi, Y., Brown, A.G., Lawford, P.V., et al., 2011, "Computational modelling and evaluation of cardiovascular response under pulsatile impeller pump support," Interface Focus, 1(3), pp 320-337. doi: 10.1098/rsfs.2010.0039.

[18] Shi, Y., Lawford, P.V., Hose, D.R., 2017, "Numerical Modeling of Hemodynamics with Pulsatile Impeller Pump Support," Ann. Biomed. Eng., 38, pp. 2621.

[19] Suga, H., 1979, "Total mechanical energy of a ventricular model and cardiac oxygen consumption," Am. J. Physiol. 236 (Heart Circ. Physiol. 5), pp. H498-H505.

[20] Suga, H., 1990, "Ventricular energetics," Physiol. Rev., 70, pp. 247-277.

[21] Suga, H., Sagawa, K., Shoukas, A.A., 1973, "Load Independence of the Instantaneous Pressure-Volume Ratio of the Canine Left Ventricle and Effects of Epinephrine and Heart Rate on the Ratio," Circ. Res., 32, pp. 314-322.

[22] Suga, H., Sagawa, K., 1974, "Instantaneous Pressure-Volume Relationships and Their Ratio in the Excised, Supported Canine Left Ventricle," Circ. Res., 35, pp. 117-126. 
[23] Klodell, C.T., Morey, E., Lobato, E.B., Aranda, J.M., et al., 2007, "Effect of Sildenafil on Pulmonary Artery Pressure, Systemic Pressure, and Nitric Oxide Utilization in Patients With Left Ventricular Assist Devices," The Annals of Thoracic Surgery, 83(1), pp. 68-71.

[24] Jaski, B.E., Fifer, M.A., Wright, R.F., Braunwald, E., Colucci, W.S., 1985, "Positive inotropic and vasodilator actions of milrinone in patients with severe congestive heart failure. Dose-response relationships and comparison to nitroprusside,",J. Clin. Invest., 75(2), pp. 643-649.

[25] Nizamic, T., Murad, M.H., Allen, L.A., Mcllvennan, C.K., Wordingham, S.E., et al., 2001, "Ambulatory Inotrope Infusions in Advanced Heart Failure," JACC: Heart Failure, 6(9), pp. 757-767.

[26] Harris, T., Hardin, J.W., 2013, "Exact Wilcoxon signed-rank and Wilcoxon MannWhitney ranksum tests," The Stata Journal, 13(2), pp. 337-343.

[27] Uriel, N., Adatya, S., Malý, J., Kruse, E., et al., 2017, "Clinical hemodynamic evaluation of patients implanted with a fully magnetically levitated left ventricular assist device (HeartMate3)," J. Heart Lung Transplant, 36, pp. 28-35.

[28] Choi, S., Boston, J.R., Antaki, J.F., 2007, "Hemodynamic controller for left ventricular assist device based on pulsatility ratio," Artif. Organs, 31(2), pp. 114-25.

[29] Topilsky, Y., Maltais, S., Oh, J.K., Atchison, F.W., et al., 2010, "Focused Review on Transthoracic Echocardiographic Assessment of Patients with Continuous Axial Left Ventricular Assist Devices," Cardiology Research and Practice, 2011, pp. 1-11.

[30] Fukamachi, K., Shiose, A., Massiello, A., Horvath, D.J., et al., 2013, "Preload Sensitivity in Cardiac Assist Devices," Ann. Thorac. Surg., 9(1), pp. 373-380.

[31] Chabiniok, R., Wang, V.Y., Hadjicharalambous, M., Asner, L., Lee, J., Sermesant, M., Kuhl, E., et al., 2006, "Multiphysics and multiscale modelling, data-model fusion and integration of organ physiology in the clinic: ventricular cardiac mechanics," Interface Focus, 6(2). doi: 10.1098/rsfs.2015.0083.

[32] Anderson, A.E., Ellis, B.J., Weiss, J.A. 2007, "Verification, validation and sensitivity studies in computational biomechanics," Comput. Methods Biomech. Biomed. Eng., 1(3), pp. 171-184.

[33] Asner, L., Hadjicharalambous, M., Chabiniok, R., Peressutti, D., Sammut, E., Wong, J., Carr-White, G., Razavi, R., King, A.P., Smith, N., Lee, J., Nordsletten, D. 2017, "Patientspecific modelling for left ventricular mechanics using data-driven boundary energies," Comput. Methods Appl. Mech. Eng., 314, pp. 269-295. 
De Lazzari Claudio

[34] Cikes, M., Solomon, S.D. 2016, "Beyond ejection fraction: an integrative approach for assessment of cardiac structure and function in heart failure," Eur. Heart J., 37, pp. 1642-1650.

[35] Katz, A.M., Rolett, E.L. 2016, "Heart failure: when form fails to follow function," Eur. Heart J., 37, pp. 449-454.

[36] Fonarow, G.C., Hsu, J.J. 2016, "Left Ventricular Ejection Fraction. What is "Normal"?," JACC Heart Fail., 4(6), pp. 511-513.

[37] Ky. B., French, B., Khan, A.M., Plappert, T., Wang, A., Chirinos, J.A., Fang, J.C., Sweitzer, N.K., et al., 2013, "Ventricular-Arterial Coupling, Remodelling and Prognosis in Chronic Heart Failure," J. Am. Coll. Cardiol., 62(13), pp. 1165-1172. 


\section{Figure Captions List}

Fig. 1 Panel [a] shows the electrical analogue of the cardiovascular system. $\mathrm{R}_{\mathrm{AT}}$, $\mathrm{R}_{T T}$ and $\mathrm{R}_{\mathrm{BT}}\left(\mathrm{C}_{\mathrm{AT}}, \mathrm{C}_{\mathrm{TT}}\right.$ and $\mathrm{C}_{\mathrm{BT}}$ ) represent the aortic, thoracic and abdominal tract resistance (compliance), respectively. $L_{A T}, L_{T T}$ and $L_{B T}$ are aortic, thoracic and abdominal tract inertance, respectively. Ras is the variable peripheral arterial resistance. $C_{v s}\left(R_{v s 1}\right.$ and $R_{v s 2}$ ) reproduces the systemic venous compliance (variable resistances). $\mathrm{R}_{\mathrm{PAM}}, \mathrm{R}_{\mathrm{PAS}}, \mathrm{C}_{\mathrm{PAM}}, \mathrm{C}_{\mathrm{PAS}}$, LPAM and LPAS are the main and small pulmonary resistances, compliances and inertances, respectively. $R_{P A R}$ and $R_{P C}$ are the pulmonary arteriole and capillary resistances. Panel $[\mathrm{b}]$ and $[\mathrm{c}]$ show the left and right heart with the valves between the atria end the ventricle and between the left (right) ventricle and the aorta (pulmonary artery). Each valve is modeled using a diode and a resistance $[4-6,15]$. In panels [a], [b], and [c] Pt represents the mean intrathoracic pressure. Panel [d] shows the electrical analogue of the LVAD. The inlet (outlet) pump cannula is modeled with resistance, inertance and compliance elements. Q $\left.\mathrm{VPI}_{\text {( }} \mathrm{Q}_{\mathrm{VPO}}\right)$ is the inlet (outlet) LVAD cannula flow. QVAD represents the LVAD flow. Pas (Plv) is the systemic arterial (left ventricular) pressure.

Fig. 2 Left ventricular P-V plane. SV is the stroke volume; EDV (ESV) is the enddiastolic (end-systolic) ventricular volume; Pes (Ped) is the end-systolic (end-diastolic) ventricular pressure; ESPVR (EDPVR) is the end-systolic 
(end-diastolic) pressure volume relationship; $E_{a}\left(E_{e s}\right)$ is the arterial (ventricular) elastance; $V_{0}$ is the zero pressure filling volume; $E W, P E$ and PVA are the external work, the potential energy and the pressure volume area respectively [19-22].

Fig. 3 Percent variation from admission patient conditions of the left ventricular external work. Upper panel shows EW percent variation when LVAD (at $6000 \mathrm{rpm}$ ) was applied without and with Milrinone administration on patients \#1, \#2 and \#3. In the lower panel the simulation results are obtained when INCOR pump was applied at 6000 rpm (patient \#4) and in conjunction with the drug injection (patients \#4 and \#5). Simulations with pump rotational speed fixed at 8900 and 10000 rpm in conjunction and without Milrinone administration, were performed for all three patients.

Fig. 4 Percent variation from admission patient conditions of the right ventricular external work. Upper panel shows EW percent variation when LVAD (at $6000 \mathrm{rpm}$ ) was applied without and with Milrinone administration on patients \#1, \#2 and \#3. In the lower panel the simulation results are obtained when INCOR pump was applied at 6000 rpm (patient \#4) and in conjunction with the drug injection (patients \#4 and \#5). Simulations with pump rotational speed fixed at 8900 and 10000 rpm in conjunction and without Milrinone administration, were 
De Lazzari Claudio

performed for all three patients.

Fig. 5 Percent variation from admission patient conditions of the left ventricular PVA. Both panels show simulation results obtained as described in Fig.2.

Fig. 6 Percent variation respect to admission patient conditions of the right ventricular PVA. Both panels show simulation results obtained as described in Fig.3.

Fig. 7 Comparison between measured and simulated left ventricular ejection fraction. 
De Lazzari Claudio

\section{Table Caption List}

Table $1 \quad$ List of symbols used in Eqs. (1) and (2)

Table 2 List of symbols used in Eq. (3) and parameter values assigned during the simulations performed with CARDIOSIM ${ }^{\circledR}$

Table 3 Hemodynamic data measured on the six patients

Table 4 Simulation results regarding the reproduction of hemodynamic parameter measured on the six patients, reported in Table 3

Table 5 Effects induced on hemodynamic parameters by the simultaneous LVAD assistance and Milrinone administration in patients \#4, \#5 and \#6. 